\title{
Probabilistic resilience assessment of civil systems: analysis and validity of the PEER framework
}

\author{
M. Broccardo, P. Galanis, S. Esposito and B. Stojadinovic \\ Institut für Baustatik und Konstruktion \\ Eidgenössischen Technischen Hochschule (ETH), Zürich, Switzerland
}

\begin{abstract}
:
The PEER framework formula, promoted and used by researchers from the Pacific Earthquake Engineering Research (PEER) Center, has been proved to be a significant step in the realization of a performance-based earthquake engineering approach. The key objective of this short study is to investigate the use of the classical PEER framework in assessing the resilience of facilities or systems against natural hazards, with a particular focus on seismic loads. The resilience is a metric that accounts for the performance of a facility or a system after a shock and during its recovery phase. It is therefore of interest to have accurate, yet simple, tools to compute statics of this metric. In this study, the PEER framework formula is first revisited by imposing the resilience of a facility as a final decision variable, after which the limitations and the range of applicability are closely analyzed. The resilience assessment framework thusly developed sets the foundation for the implementation of resilient management strategies which can address optimal targets for a given hazard. A simple example with fully ergodic uncertainties approximation is introduced to practically show the computation of resilience statistics, such as the mean annual rate, the complementary cumulative distribution function, the mean annual cumulative value, and the approximate probability of extremes. Finally, we introduce the concept of a "resilience breaking point", which represents scenarios that lead to the point where the system stops being resilient. The resilience breaking points, as well as the potential events that could lead to such points, are of particular importance, as they define the critical scenarios that should be taken into meticulous account by policy makers. It is shown that the framework validity bounds can be used to define the range in which some of these critical points may occur.
\end{abstract}

\section{INTRODUCTION}

Performances of human systems are strongly affected by natural disasters such as earthquakes, floods, droughts, storms, etc. The development of mathematical and statistical models to assess the risk arising from different natural hazards is essential towards creating and managing resilient societies. Using performance-based designs and analysis is a desirable practice for reducing and managing these risks. In the domain of earthquake engineering, scholars and researchers at the Pacific Earthquake Engineering Research Center (PEER) have applied the PEER framework formula in order to develop such performedbased analysis. The formula has been originally proposed by Cornell \& Krawinkler (2000), and later has been used and refined in different studies to estimate the statistics of different performance metrics, see Moehle \& Deierlein (2004) and Mackie \& Stojadinovic (2001). The PEER framework has later been employed to estimate the mean rate of performance loss for complex systems through simulations. Ex- amples of this include Jayaram \& Baker (2010) and Esposito (2011). In recent years, the resilience, or its loss, has been proposed as a performance metric for facilities or systems. In a renowned paper, Bruneau et al. 2003 conceptualized the resilience as a metric that "can be understood as the ability of the system to reduce the chances of a shock, to absorb a shock if it occurs, and to recover quickly after a shock". Following Bruneau et al. pioneering study, considerable attention has been recently devoted towards developing frameworks to assess the resilience of civil facilities or infrastructures; among these, notable are, Bocchini et al. 2014, Cimellaro et al. 2010. Within the burgeoning literature in this field, the key contribution of this study is to investigate the use of the PEER formula in computing the statistics of the resilience metric. Our study commences by closely analyzing the range of applicability of the framework developed in the PEER center in the context of resilience. In particular, we show that the range of applicability of this framework depends on the interaction between the recovery time and the inter-arrival time of a future event. Finally, 
the concept of a "resilience breaking point", which, in short, represents scenarios that lead to the point where the system ceases being resilient, is introduced for the given virtual region. With these resilience breaking points, we enable policy makers to identify critical scenarios that should be taken into meticulous account in policy planning and execution. We show that a particular set of resilience breaking points may originate from possible interactions between the recovery phase and the occurrences of new events. The "applicability" criteria used to define the range of the validity of the resilience PEER framework can then be used to prevent the materialization of such events, and thus to positively impact the performance of human systems.

\section{THE PEER FORMULA IN THE RESILIENCE CONTEXT}

The classical performance-based earthquake engineering approach builds on the well-known PEER formula, i.e.,

$$
\begin{aligned}
\lambda(d v)= & \int_{d} \int_{e d p} \int_{i m} G(d v \mid d)|d G(d \mid e d p)| \\
& |d G(e d p \mid i m)||d \lambda(i m)|
\end{aligned}
$$

where $i m$ is an intensity measure (e.g., peak ground acceleration, peak ground velocity, spectral acceleration, etc.), edp is an engineering demand parameter (e.g., interstorey drift), $d$ is a damage measure (e.g., minor, medium extensive, etc.), $d v$ is a decision variable (e.g., monetary losses, downtown time, etc.), $\lambda(x)$ is a mean annual rate of events exceeding a given threshold for a given variable $x$, and $G(y \mid x)=$ $P(Y \geq y \mid X=x)$ is a conditional complementary cumulative distribution function (CCDF). Note that, in general, $d$ is a discrete variable so that $\sum_{d}$ substitutes $\int_{d}$. Moreover, the variables $i m$, $e d p$, and $d m$ can be expressed in vector form; the integral then involves several folds. The PEER framework was originally presented by Cornell \& Krawinkler (2000).

In recent years, the "loss of resilience", here named $r$, has been proposed as the metric of post event performance for facilities and/or networks. The loss of resilience essentially accounts for the loss of facility performance during the recovery time. In the PEER framework, it thus appears logical to impose $r \equiv d v$. The $r$ is an area defined as

$$
r=\int_{0}^{\tau} \widehat{r f}(t) d t=\tau \times a \times c(a),
$$

where $\widehat{r f}(t)$ is the recovery function defined in the interval $[0, \tau], a=\max _{[0, \tau]} \widehat{r f}(t)$ is the performance drop (absorption phase, which depends on damage $d$ ), and $c$ is a constant dependent on the shape of $\widehat{r f}(t)$. The constant $c$ is characteristic of the facility and the recovery strategies, and, in this context, we assumed it to be dependent on the performance drop $a$. Both $\mathcal{T}$ and $A$ are considered random variables (thus denoted with an upper case), while $c$ is considered a deterministic mapping. We will soon return to the assumptions underlying these choices. We can finally write

$$
\mathcal{R}=\mathcal{T} A c(A),
$$

and investigate the statistics of the resilience loss $\mathcal{R}$. In particular, we are interested in the CCDF of $\mathcal{R}$, its mean annual rate $\lambda(r)$, and the probability of its extreme given a time span. To integrate (3) into the PEER formulation, we make our first assumption that $A$ is conditionally independent from the variables $E D P, I M$ given $D=d$. Thus, as $A \Perp$ $E D P, I M \mid D=d$, we can first define $G_{A \mid d}(a \mid d)$, and then obtain the CCDF of $A$ as

$$
\begin{array}{r}
G_{A}(a)=\int_{d m} \int_{e d p} \int_{i m} G_{A \mid d}(a \mid d)\left|d G_{D \mid e d p}(d \mid e d p)\right| \\
\left|d G_{e d p \mid i m}(E D P \mid i m)\right|\left|d G_{i m}(i m)\right| .
\end{array}
$$

Our second assumption is that the recovery time $\mathcal{T}$ is statistically independent from all the other variables given a performance $\operatorname{drop} a$, that is, $\mathcal{T} \Perp$ $D, E D P, I M \mid A=a$. Our third fundamental assumption is that the recovery time $\mathcal{T}$ is "much shorter" than the intra-arrival time of an event, $T_{i n}$, for a given $I M>i m$ of interest, that is, $P\left(\mathcal{T}>T_{i n} \mid I M>i m\right)<$ $\epsilon$ for a sufficiently small $\epsilon$. Given the first two assumptions, we can write the joint CCDF of $A$ and $\mathcal{T}$ as $G(\tau, a)=G_{\mathcal{T} \mid a}(\tau \mid a) G_{A}(a)$ for a prescribed recovery distribution $G_{\mathcal{T} \mid a}(\tau \mid a)$, and then derive the statistics of $\mathcal{R}$. We are particularly interested in transforming the conditional distribution of $\mathcal{T}$ given $A=a$ into the conditional distribution of $\mathcal{R}$ given $A=a$, that is, $P(\mathcal{R}>r \mid A=a)=P(\mathcal{T} a c>r \mid A=a)=P(\mathcal{T}>$ $r /(a c) \mid A=a)$, which is equivalent to

$$
G_{\mathcal{R} \mid a}(r \mid a)=G_{\mathcal{T} \mid a}\left(\frac{r}{a c(a)} \mid a\right) .
$$

Given (5), we can finally obtain the CCDF of $\mathcal{R}$, which is simply

$$
G_{\mathcal{R}}(r)=\int_{a} G_{\mathcal{T} \mid a}\left(\frac{r}{a c(a)} \mid a\right)\left|d G_{A}(a)\right|,
$$

and the mean rate $\lambda(r)$ by rewriting the PEER formula as

$$
\begin{array}{r}
\lambda(r)=\int_{a} \int_{d} \int_{e d p} \int_{i m} G_{\mathcal{T} \mid a}\left(\frac{r}{a c(a)} \mid a\right)\left|d G_{A \mid d}(a \mid d)\right| \\
\left|d G_{D \mid e d p}(d \mid e d p)\right|\left|d G_{E D P \mid i m}(e d p \mid i m)\right||d \lambda(i m)| .
\end{array}
$$

This is an important relationship since for a defined recovery distribution $G_{\mathcal{T} \mid a}$ and shape of the recovery function $\widehat{r f}(t)$, it directly produces the mean rate of 
resilience loss $\lambda(r)$. To the best of our knowledge, (7) has not been applied before. Another key metric is the expected annual cumulative resilience loss. As pointed out by (Der Kiureghian 2005), this quantity can be written as

$$
\begin{aligned}
E\left[\sum \mathcal{R}\right] & =\int_{0}^{\infty} r|d \lambda(r)|=[r \lambda(r)]_{0}^{\infty}+ \\
& +\int_{0}^{\infty} \lambda(r) d r=\int_{0}^{\infty} \lambda(r) d r
\end{aligned}
$$

where the last equality is given by the fact that $\lim _{r \rightarrow \infty} r \lambda(r) \rightarrow 0$. This condition is satisfied if, for example, $\lambda(r)$ has an exponential decay, and it commonly holds if our third assumption, that is, $P(\mathcal{T}>$ $\left.T_{i} n\right)<\epsilon$, is met.

\section{UNDERSTANDING THE ASSUMPTIONS}

There are three fundamental assumptions underlying (7):

\section{1. $A \Perp E D P, I M \mid D=d$ \\ 2. $T \Perp D, E D P, I M \mid A=a$}

3. $P\left(\mathcal{T}>T_{\text {in }} \mid I M>i m\right)<\epsilon$ for a sufficiently small $\epsilon$.

The first assumption is "robustly" consistent with the PEER framework. The same argument holds for the second assumption, although here one can argue that it is a weaker statement, since the recovery time depends also on external factors, such as the performance drops of the other facilities and infrastructures. These assumptions share the limitations of the PEER framework from which they have been extracted. Nevertheless, it is important to recognize that they are enablers of a manageable and realistic framework, the benefits of which outweigh the usual reservations that emanate from abstractions. Assumption 3 is the most delicate one, since it defines the range of applicability of (7). This condition states that, given an intensity range of interest $I M>i m$, the probability that the recovery time is greater than the intraarrival time of a given event, is "negligible". To compute this probability, we introduce a fourth assumption:

\section{4. $\mathcal{T} \Perp T_{\text {in }} \mid I M>i m$.}

This is a reasonable assumption, since for a given $I M>i m$, the processes that govern the recovery time are "independent" from the arrival of the next event of intensity $I M>\mathrm{im}$. Assuming that the occurrence of events, such earthquakes, follows a Poisson distribution, the intra-arrival time between two events, which is equivalent to the first arrival time, follows an exponential distribution with mean rate of occurrence $\lambda(\mathrm{im})$, i.e., $T_{\text {in }} \sim \exp (\lambda(\mathrm{im}))$, while the CCDF of the recovery time is given by

$$
G_{\mathcal{T}}(\tau)=\int_{\tau} G_{\mathcal{T} \mid a}(\tau \mid A=a) G(A \mid I M>i m) .
$$

Note that while, for simplicity, we did not explicitely write $G_{\mathcal{T}}(\tau \mid I M>i m)$, it is assumed implicitly in the notation $G_{\mathcal{T}}(\tau)$. From now, we will thus drop the implicit condition $I M>i m$. Following this, we can write $P\left(\mathcal{T}>T_{\text {in }}\right)=P\left(\mathcal{T}-T_{\text {in }}>0\right)$, which can be derived by defining $\bar{T}=\mathcal{T}-T_{\text {in }}$ and computing $P\left(\mathcal{T}-T_{\text {in }}>0\right)=G_{\bar{T}}(0)$. Since $G_{\bar{T}}(\bar{t})$ is simply

$$
\begin{aligned}
G_{\bar{T}}(\bar{t}) & =\left(G_{\mathcal{T}} * f_{T_{i n}}(-\cdot)\right)(\bar{t}) \\
& =\int_{0}^{\infty} f_{T_{i n}}(t-\bar{t}) G_{\mathcal{T}}(t) d t
\end{aligned}
$$

we can write that

$$
P\left(\mathcal{T}>T_{\text {in }}\right)=\int_{0}^{\infty} f_{T_{\text {in }}}(t) G_{\mathcal{T}}(t) d t .
$$

Note that (11) can be inserted in the PEER formula framework by substituting $G_{\mathcal{T}}(t)$ for (9) and $G_{A}$ for (4). Alternatively, using elementary probability frequentist reasoning, we can obtain $G(\tau)=$ $\lambda_{\tau}(\tau) / \lambda_{\tau}(0)$.

\section{APPLYING THE PEER FRAMEWORK}

In this section, we present an application to investigate the use and the limitation of Equations (7) and (8). In order to not dwell on details, we contract the (7) and replace it with a CCDFs. The CCDFs we use is based on the experience in developing similar CCDFs using the three-step process implied by the PEER formula framework, thus it does not detract form the generality of the proposed framework. We thus want to define a direct relationship between the intensity measure $I M$ and the performance $\operatorname{drop} A$. This means that (7) can be rewritten as

$$
\begin{array}{r}
\lambda(r)=\int_{a} \int_{i m} G_{\mathcal{T} \mid a}\left(\frac{r}{a c(a)} \mid a\right) \\
\left|d G_{A \mid i m}(a \mid i m)\right||d \lambda(i m)| .
\end{array}
$$

A full example is beyond the scope of this study and will be examined in a future one. Suppose an annual hazard curve, $H(i m, 1)$, is given by the following equation

$$
H(i m, 1)=0.015 \exp (-i m(3.5+0.02 * i m)) .(13
$$

Figure 1 shows a plot of this curve. As a reminder, the hazard curve is defined as

$$
\begin{aligned}
H(i m, t) & =P[I M>i m \text { in } t \text { years }] \\
& =1-\exp \left[-G_{I M}(i m) \nu t\right] .
\end{aligned}
$$


Then, for our value of interest, we have $\lambda(\mathrm{im}) \sim$ $H(i m, 1)$. Notice that $H(0,1) \simeq \nu$, where $\nu$ is the mean rate of earthquake greater than a minimum significant value, $m>m_{\min }$. Moreover, we assume that the performance drop has a value of 100 units per day, where 0 stands no variation in performance and 100 fully not operational. The random variable $A$ thus has a finite support $[0,100]$. We define the performance drop distribution, $G_{A \mid i m}$, following a beta distribution, that is, $A \mid i m \sim \operatorname{Beta}(\alpha(\mathrm{im}), \beta(\mathrm{im}))$. The choice of the beta distribution is driven by the compact support of $A$. Following this, we set:

$$
\begin{aligned}
& \mu_{A \mid i m}(i m)=0.479 i m+0.0021 \\
& \delta_{A \mid i m}(i m)=-0.137 i m+0.314
\end{aligned}
$$

where $\delta_{A \mid i m}(\mathrm{im})$ is c.o.v.. Then, the parameters $\alpha(\mathrm{im})$ and $\beta(\mathrm{im})$ are simply given by the following relationships

$$
\begin{aligned}
& \alpha(i m)=-\frac{\mu\left(\sigma^{2}+\mu^{2}-\mu\right)}{\sigma^{2}}(i m), \\
& \beta(i m)=\frac{\left(\sigma^{2}+\mu^{2}-\mu\right)(\mu-1)}{\sigma^{2}}(i m),
\end{aligned}
$$

where $\mu \equiv \mu_{A \mid i m}(i m)$ and $\sigma \equiv \delta_{A \mid i m}(i m) \mu_{A \mid i m}(i m)$. Figure 2 shows the distribution of $G_{A \mid i m}(a \mid i m)$ for different intensity values $i m$. We notice that while the intensity $i m$ is increasing, the CCDF is shifting to the right, giving a high probability of performance drops.

Next, we assume that the recovery time distribution given a performance loss follows a lognormal distribution, i.e., $\mathcal{T} \mid a \sim \ln \mathcal{N}\left(\mu_{\mathcal{T} \mid a}(a), \mu_{\mathcal{T} \mid a}(a) \delta_{\mathcal{T} \mid a}(a)\right)$, where the parameters are assumed to trail the following relationships

$$
\begin{gathered}
\mu_{\mathcal{T} \mid a}(a)=10 \exp (0.047 a)-5 \\
\delta_{\mathcal{T} \mid a}=-0.002 a+0.310
\end{gathered}
$$

In this case, we have assumed that the mean recovery time follows an exponential law, since it is reasonable

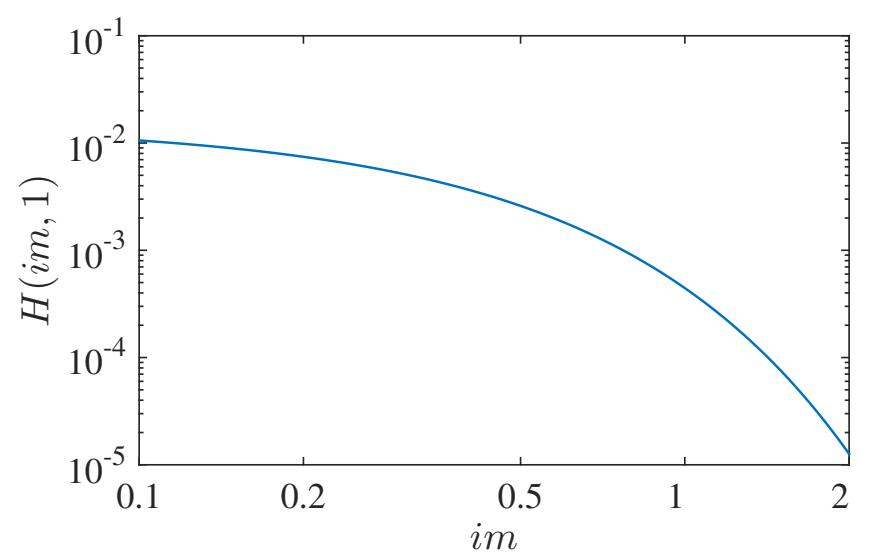

Figure 1: Assumed annual seismic hazard curve to expect a much longer restoration of heavily damaged structures compared to lightly damaged ones. Moreover, for a performance drop close to 100, the recovery time distribution has to be understood as a time of reconstruction of the facility. Figure 3 shows the distribution of $G_{\mathcal{T} \mid i m}(\tau \mid i m)$. Notice that these distributions give the probability that the structure returns to fully operational mode.

Another important factor in (12) is the definition of the "area factor" $c(a)$. In fact, since the resilience is an area, it strongly depends on the shape of the recovery function. There are systems that show a fast recovery in the first phase, so that the derivative of the recovery function is "steep", while there exist systems for which the recovery function shows a slower first phase, so that the derivative of the recovery function is "flat". Moreover, the function needs to have a compact support $[0 \tau]$. The shape of the recovery function depends on the (seismic) design of the facility and the (seismic) recovery strategies. Thus, defining the recovery function should be one of the seismic design objectives, as well as one of the principles for governing the recovery process. In this study, we decided to use a single parametric function to describe the recovery process in order to keep the model as simple, yet as flexible enough to capture the main trend. The parametric function that we will use is the following

$$
\left.\hat{r f}(t)=a\left(\frac{t}{\tau}\right)^{p}, \text { with } p \in\right] 0,+\infty[
$$

Given (21), the $c$ factor is simply

$$
c=\frac{1}{\tau a}\left(\tau a-\int_{0}^{\tau} a\left(\frac{t}{\tau}\right)^{p} d t\right)=\frac{p}{p+1} .
$$

Figure 4 shows different recovery functions for different $p$ values and the evolution of $c$ as a function of $p$.

Notice that the value of $p$ can be viewed as a resilience index for a given performance drop and a given recovery time. Higher values of $p$ indicate higher resilience losses and systems that do not have a "rapid" recovery phase. In other words, a resilient system can be defined by low values of the index $p$ for

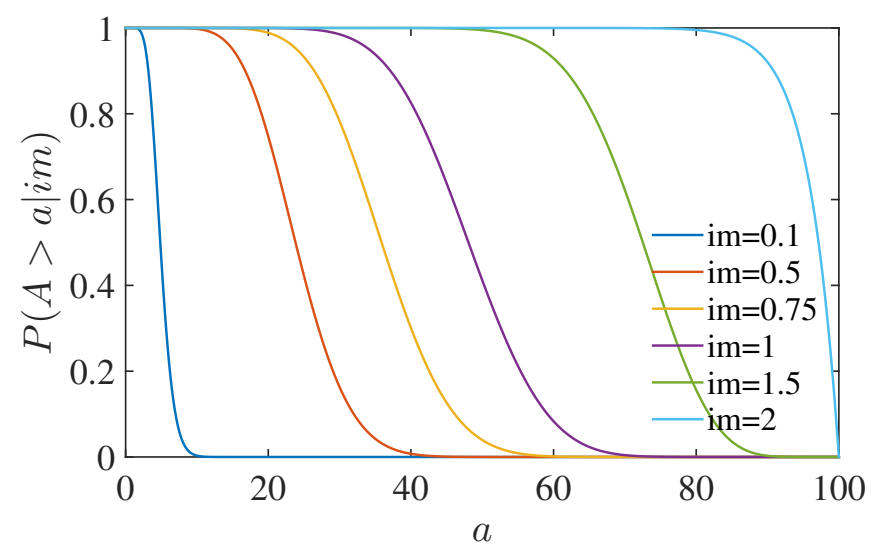

Figure 2: Performance drop distributions $G_{A \mid i m}(a \mid i m)$ 
a given performance drop $a$ and for a given recovery time $\tau$. The $p$ value can be understood as a property of the facility and of the recovery strategies, or more generally as dependent on the performance drop $a$. It is thus reasonable to assume that low values of $a$ correspond to low value of $p$ and, indeed, of $c$. We can finally write $c(a)=p(a) /(1+p(a))$ for a given $p(a)$ function.

Although the model is quite simple and $p$ allows only for one shape of the recovery function, it can be noticed that for any given general (and thus real) recovery shape, it corresponds to one unique $p$ or $c$ (obviously the inverse mapping does not hold). Given this, we can study the statistics of $p$ and $c$ considering a particular performance drop and then define a new random variable. However, in doing that, (7) will not hold, since the Markov chain underlying the PEER framework will not have a single parent-child relationship.

Assume that we want to study three facilities that share the same absorption and recovery functions defined above, but with three different $p$ values. Facility $\mathrm{A}$ and $\mathrm{B}$ have constant $p$ values of $1 / 2$ and 2 , respectively, while the facility $\mathrm{C}$ has a $p$ value that varies according to the following equation

$$
p(a)=0.018 a+0.412 \text {. }
$$

These facilities can be thought of as belonging to the following categories: A) systems that have a high rate of recovery independent from the performance drop; B) systems that have a slow rate of recovery independent form the performance drop; and C) systems that have an intensity-dependent recovery rate such that for larger performance drops the rate of recovery is lower. The three facilities considered here can be interpreted as having the same loss of performance after the event occurs and as having the same recovery time, but with three different initial rates of recovery.

Given this outline, we can compute the mean annual rate of the resilience loss for the different facilities. Figure 5 shows the three curves for the three facilities. It is of interest to observe that for a high $\lambda(r)$, the facility $\mathrm{C}$ has a loss of resilience close to facility

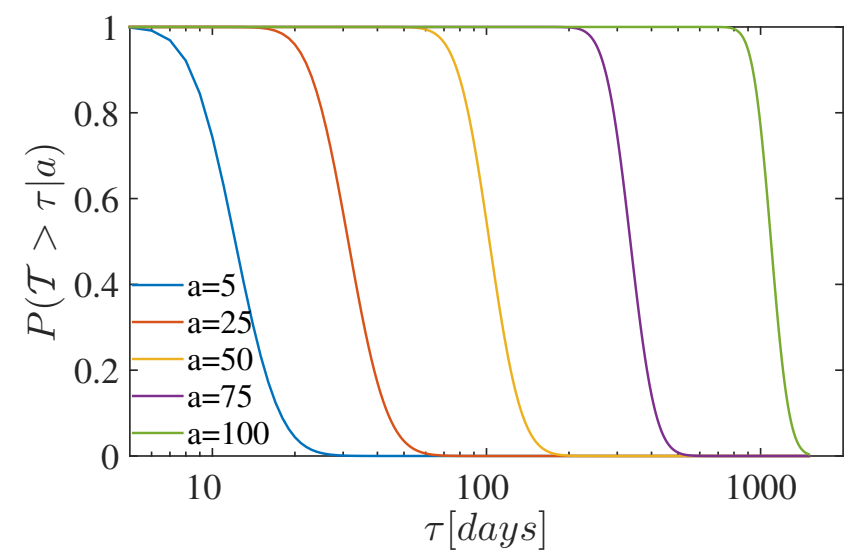

Figure 3: Recovery time distributions $G_{\mathcal{T} \mid a}(\tau \mid a)$
A, while for a low $\lambda(r)$, it has losses close to facility $\mathrm{B}$, and this is because its rate of recovery depends on $A$. As a final step, we are able to compute the cumulative resilience loss by using (8). Table 4 shows the results of this computation for the three facilities.

Table 1: Cumulative loss of resilience for the three facilities

\begin{tabular}{ccc}
\hline Facility type & $p$ & $E\left[\sum \mathcal{R}\right]$ \\
\hline A & 0.5 & 3.617 \\
B & 2.0 & 7.169 \\
C & $0.018 \mathrm{a}+0.412$. & 6.159 \\
\hline
\end{tabular}

\subsection{Probability of extremes and ergodicity of the framework}

In this subsection, we compute the probability of maximum resilience loss for given $t$ years. Note that the probability of extremes, which is the probability that at least one event over a series of events in $t$ years is greater than a given threshold, i.e., $P[\mathcal{R}>$ $r$ in $t$ years], is different from the CCDF, which is the probability that a random event is greater than a given threshold, i.e., $P[\mathcal{R}>r]=G_{\mathcal{R}}$. If all the intermediate variables defining $\lambda(r)$ are ergodic and thus satisfy the underlying assumption of a Poisson process, then we can write

$$
\begin{aligned}
\bar{H}(r, t) & =P[\mathcal{R}>r \text { in } t \text { years }] \\
& =1-P[0 \text { events } \mathcal{R}>r \text { in } t \text { years }] \\
& =1-\exp [-\lambda(r) t] .
\end{aligned}
$$

However, it has been pointed out by Der Kiureghian (2005) that the intensity variables $I M$ s have uncertainties that renew at each occurrence of an earthquake, while the "structural variables", such as EDPs and $D \mathrm{~s}$, are also characterized by timeinvariant uncertainties. More generally, the occurrence of these latter variables for different earthquakes cannot be considered statistically independent. In other words, the ergodic assumption is valid

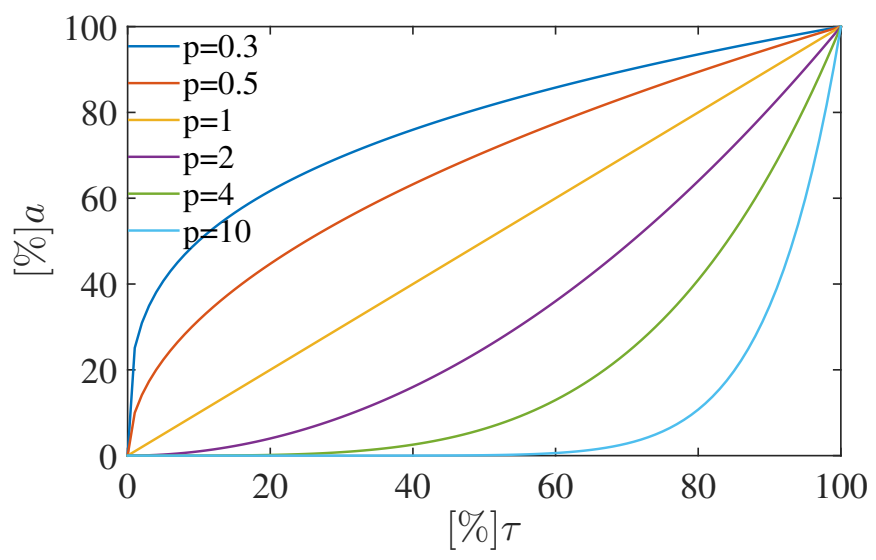

Figure 4: Recovery functions $\widehat{r f}(t)$ 
only for the intensity measures, while it is generally invalid for the other variables involved (Der Kiureghian \& Ditlevsen 2009). The (24) can commonly be shown to be only an upper bound of the true $H(r, t)$ (Der Kiureghian 2005). This is the reason why we use the overline in (24). For a given intensity measure $I M=i m$, the total uncertainty in EDP or in other variables can be thought of as the combination of an ergodic component and a non-ergodic component.

Notice that, the correct solution can only be found by a clear classification of the uncertainties during the analysis. However, this process can be very difficult or even impossible in the PEER framework if the intermediate distributions (vulnerability and recovery distributions) are "given" or "prescribed". We will now provide a small example to support this statement. Suppose that the performance drop $A$ is related to the intensity of the earthquake via the following simple linear model

$$
A=\mathcal{M}(I M, \Theta)=I M \Theta
$$

where $\Theta$ is the "response" of the facility for a unit change in $I M$ and $\Theta \Perp I M$. Clearly, $\Theta$ is an inherent property of the structure with uncertainties that do not renew over different occurances of events, and it is thus non-ergodic. The random variable $A$ is hence the composition of an ergodic and non-ergodic random variable. Suppose that we are interested in $P[A>a$, in $t$ years $]$, then the correct solution is obtained by considering the event as conditioned to the non-ergodic variables, i.e., $[A>a \mid \Theta=\theta]$. Undoubtedly, this event is ergodic since its uncertainties depend only on $I M$, and it can thus be viewed as Poisson with rate $P[A>a \mid \theta] \nu$. Following this, it is easy to show that

$$
P[A>a \mid \theta]=G(a \mid \theta)=G_{I M}\left(\frac{a}{\theta}\right),
$$

and

$$
\begin{aligned}
& P[A>a \mid \theta, \text { in t years }] \\
& =1-\exp \left[-\nu G_{I M}\left(\frac{a}{\theta}\right) t\right] .
\end{aligned}
$$

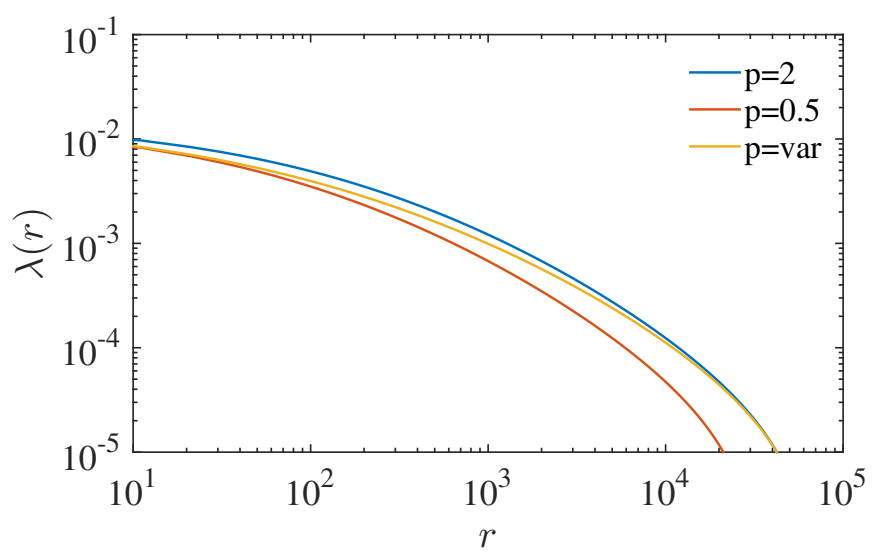

Figure 5: Mean annual rate of resilience $\widehat{r f}(t)$
The final unconditioned solution is given by the total probability theorem as

$$
\begin{aligned}
& H_{A}(a, t)=P[A>a, \text { in t years }]= \\
& =1-\int_{\theta} \exp \left[-\nu G_{I M}\left(\frac{a}{\theta}\right) t\right]\left|d G_{\theta}(\theta)\right| .
\end{aligned}
$$

This solution is naturally different from $\bar{H}_{A}(a, t)$, which assumes the ergodicity of $\theta$. However, if we apply the PEER framework to this problem, we will write the formula as $\lambda_{a}=\int_{a} G(a \mid i m)|d \lambda(i m)|$ for a prescribed $G(a \mid i m)$. We can observe in our example that the event $[a \mid I M=i m]$ is non-ergodic and that

$$
P[A>a \mid i m]=G(a \mid i m)=G_{\theta}\left(\frac{a}{i m}\right) .
$$

Now suppose that the "correct" $G(a \mid i m)$ has been prescribed without knowledge of the given model $\mathcal{M}(I M, \Theta)$, which is the case when we select predefined vulnerability functions or empirical fragility functions. As we can see, it is then impossible to derive the correct formula (28) without knowing the relationship between $A$ and $I M$, i.e., $\mathcal{M}(I M, \Theta)$, and the classification underlying the uncertainties. As Der Kiureghian (2005) shows, the $\bar{H}(\cdot, t)$ is generally an upper bound to the true $H(\cdot, t)$. It worthwhile to investigate a formulation that finds a lower bound, i.e., $\underline{H}(\cdot, t)$, in future studies.

Figure 6 shows the upper bound $(\bar{H}(r, t))$ for the three systems over a time span of 50 years.

\subsection{Validity of the framework}

In this subsection, we will look at the validity of the framework for the resilience of our example. The original PEER framework (1) assumes that CCDFs of the intermediate variables are time invariant, which implies two fundamental assumptions: a) the structural performance of the facility is time invariant, i.e, not deteriorating; and b) the structure fully recovers to its original state and performance immediately after an event. Clearly, the latter assumption suggests a

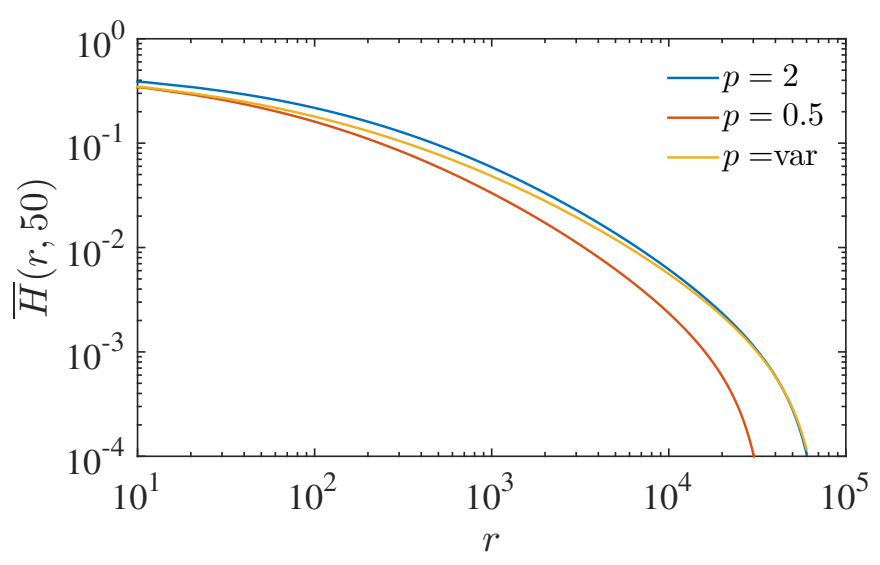

Figure 6: Probability of of $\mathcal{R}>r$ in 50 years 
recovery time equal to zero, or, in practical terms, that the recovery is sufficiently fast so as to not interact with the next event. As we have seen in section 3, this entails in probabilistic terms that $P\left[\mathcal{T}>T_{i n} \mid I M>\right.$ $m] \rightarrow 0$. We essentially need to define a sufficient small $\epsilon$ so that (7) can be considered valid. The exact solution of this problem is given by (10); in our example, we obtain $P\left[\mathcal{T}>T_{\text {in }} \mid I M>0\right]=7.0366 \cdot 10^{-4}$.

Notice that this has to be understood as the probability that for a given random earthquake, the recovery time is longer than for the next random earthquake. One may doubt this metric since it considers all events and is not conditioned on the extremes. An alternative way to think about this problem is to assume that an event leading to a large loss of performance has occurred. In that case, the probability distribution of the recovery time is simply $G_{\tau \mid A}(\tau \mid A>$ $\left.a_{\max }\right)$, which can interpreted as the reconstruction time distribution. Following this, the probabilistic question we want to address here is: what is the probability that another earthquake occurs before the structure is fully operational? If this probability is small enough, then we can consider the framework valid. Or, formulated mathematically:

$$
P\left(\mathcal{T}>T_{\text {in }} \mid A>a_{\text {max }}\right)<\hat{\epsilon} .
$$

$T_{i n}$ is again the inter-arrival time for a given $I M>$ $\mathrm{im}$. Note that, here, after we condition on the extreme event $A>a_{\max }$, we can freely choose a significant intensity that can cause the performance drop during the recovery phase. To compute (30), we assume that

$$
\mathcal{T} \Perp T_{\text {in }}(i m) \mid A>a_{\max } .
$$

This is a robust assumption, since the recovery time, given an event has occurred, is statistically independent from the arrival of the next earthquake. Following this, we can simply rewrite (11) as

$$
\begin{aligned}
& P\left(\mathcal{T}>T_{\text {in }} \mid A>a_{\text {max }}\right) \\
& =\int_{0}^{\infty} f_{T_{\text {in }} \mid \text { im }}(t) G_{\mathcal{T} \mid a}\left(t \mid A>a_{\max }\right) d t
\end{aligned}
$$

Figure 7 shows the numerical values of (32) as a function of the selected $i m$ for the inter-arrival time. The acceptance limits $\epsilon, \hat{\epsilon}$ are not defined in this study, and thus pose limits to our framework when compared to one that can consider interactions.

\section{RESILIENCE BREAKING POINTS}

When the interactions between the recovery time and the inter-arrival time have significant probabilities of occurance, then the framework (7) is invalid. However, it should be recognized that the validity criteria introduced in section 3 and 4.2 could also be considered as measures of the resilience of a system, in particular, for extreme events and scenario-based designs. In these cases, it is important to identify events that lead to large, and thus non-acceptable losses of resilience, for a defined time span. When an event or a series of events generates such losses of resilience, we characterize the system as not resilient. We can define these events as "resilience breaking points" and their formal definition is as follows:

Resilience breaking points represents scenarios that lead to the point where the system stops being resilient.

The resilience breaking points, as well as the potential events that could lead to such points, are of particular importance, as they define the critical scenarios that should be taken into meticulous account by policy makers. To define a resilience breaking point, we first require a time span that defines the time scale. For example, this can be the expected life of an individual or a standard time imposed by policy makers. Additionally, we need an acceptance criteria in terms of resilience loss. Note that once the selected time is defined, the resilience loss can be expressed in percentage over the full loss of resilience. To identify such events, both the recovery time and the rate of recovery play a key role. Some resilience breaking points are easy to recognize: they are events that have a long recovery time and a low rate of recovery. Nuclear meltdowns and contaminations of areas are classical resilience breaking point examples. The prolonged recovery time (compared to the expected lifespan) after the 1986 Chernobyl and the 2011 Fukushima accidents are sufficient to classify them as recovery breaking points. The key issue in these cases is not their classification, but the assessment of their probability, assuming it determinable. However, not all resilience breaking points are easily to identify, in particular when we have complex systems. This is compounded by the generation of resilience breaking points by the possible interaction between the recovery phase and future events, or, to paraphrase, when the aforementioned framework is invalid. Conversely, if the system or the facility satisfies the validity criteria of the framework, then the resilience breaking points will

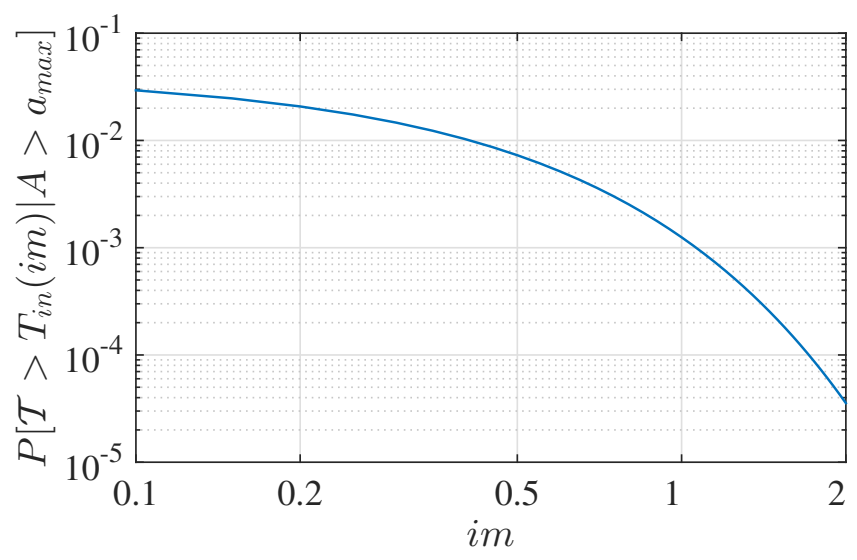

Figure 7: Probability that reconstruction time is larger than the inter-arrival time 


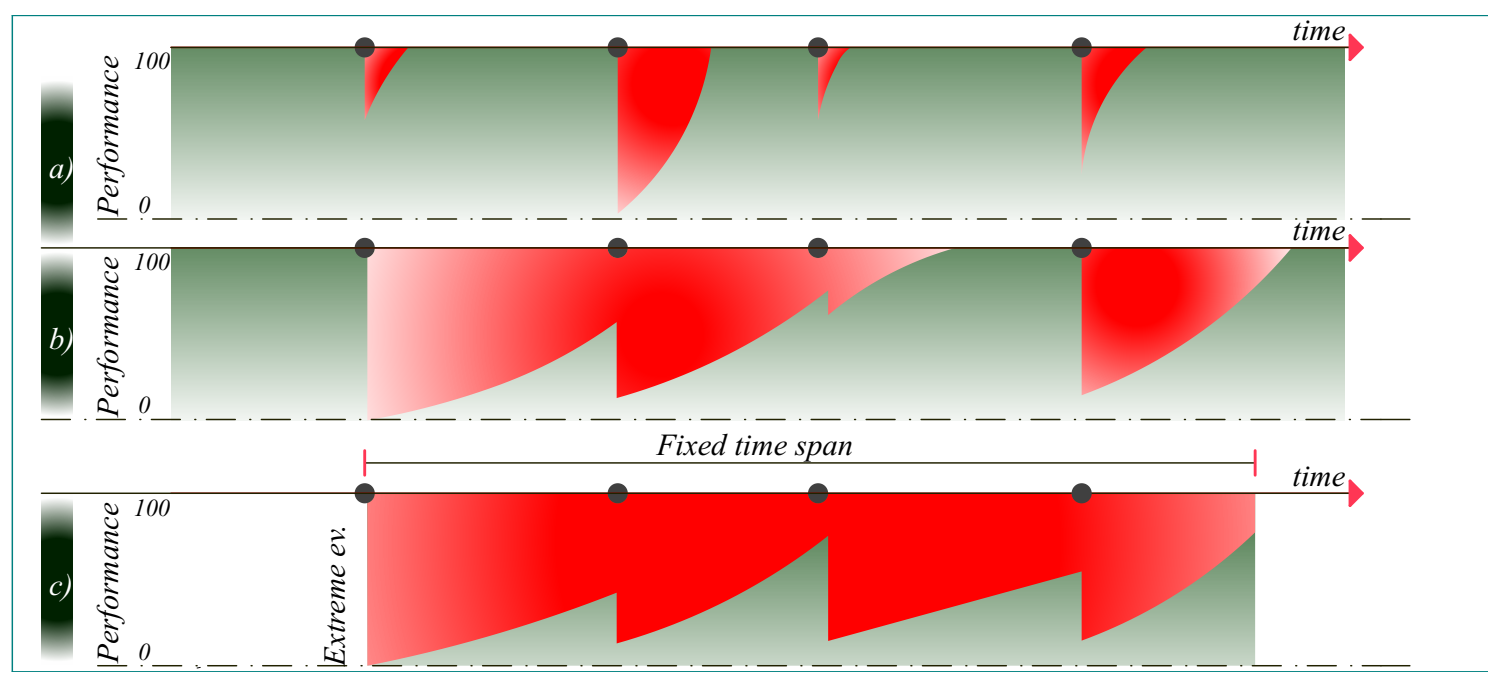

Figure 8: Examples of interactions between the recovery and the occurrence of events. a) Interaction between recovery time and interarrival time is negligible, i.e., (7) can be applied; b) Interaction between recovery time and inter-arrival time is significant, i.e., (7) cannot be applied; c) Example of resilience breaking point due to interaction of events

not occur. Figure 8.a) shows a sample of a stochastic process for which the interaction between consecutive events is negligible. Here, the claim that no resilience breaking point can occur is appropriate. Figure 8.b) shows a sample of a stochastic process for which the interaction between consecutive events is significant. Then, (7) is invalid and a resilience breaking point may occur. Finally, figure 8.c) shows a sample of a resilience breaking point: first, an extreme event occurs, after which the system has an unacceptable level of resilience loss over a selected time span.

When the validity of our framework is in doubt, models used in time variant reliability problems for degrading structures can be "modified" to describe the interaction. As an example, consider the following compound process

$$
A(t)=\sum_{n=1}^{N(t)} A_{n} \widehat{r f}(t) H\left(t-T_{n}\right),
$$

where $A_{1}, A_{2}, \ldots$ are decrements of resilience for a given event, $N(t)$ is a Poisson with mean rate $\lambda(\mathrm{im})$, $H\left(t-T_{n}\right)$ is the Heavyside step function, and $T_{n}$ the inter-arrival between events. Figure 8.c) presents a sample of this stochastic process. Moreover, if $\widehat{r f}(t)$ are exponentials, i.e., $\widehat{r f}(t)=\exp \left(\eta\left(t-T_{n}\right)\right)$ and the $A_{n} s$ are statistically independent, then, a close form solution of (33) can be analytically derived by the method of moments and thus also its integral over time. In a future work, the statistics of this or similar models will be derived and compared against (7) to investigate the magnitude of acceptance $\epsilon, \hat{\epsilon}$.

\section{CONCLUSION}

In this study, we set out to extend the PEER framework formula in order to account for the loss of resilience for a given civil facility or system. It has been shown that for a given conditional performance drop distribution, a conditional recovery time distribution, and a recovery function, the resilience can be easily integrated in the original Markovian framework. The limitations of our framework have been studied by analyzing the probability of interaction between the recovery time and the inter-arrival time of seismic events. Finally, we introduced the concept of resilience breaking point events, for which we have shown that their occurrence can be controlled with a certain confidence interval obtained using the limitation criteria of the PEER framework.

\section{REFERENCES}

Bocchini, P., D. M. Frangopol, T. Ummenhofer, \& T. Zinke (2014, June). Resilience and sustainability of civil infrastructure: Toward a unified approach. J. Infrastruct. Syst. 20(2).

Bruneau, M., S. E. Chang, R. T. Eguchi, G. C. Lee, T. D. O'Rourke, A. M. Reinhorn, M. Shinozuka, K. Tierney, W. A. Wallace, \& D. von Winterfeldt (2003, November). A framework to quantitatively assess and enhance the seismic resilience of communities. Earthq. Spectra 19(4), 733-752.

Cimellaro, G. P., A. M. Reinhorn, \& M. Bruneau (2010). Framework for analytical quantification of disaster resilience. Engineering Structures 32(11), 3639-3649.

Cornell, C. A. \& H. Krawinkler (2000). Progress and challenges in seismic performance assessment. PEER Center News 3(2), $1-3$.

Der Kiureghian, A. (2005, November). Non-ergodicity and peer's framework formula. Earthq. Eng. Struct. Dyn. 34(13), 1643-1652.

Der Kiureghian, A. \& O. Ditlevsen (2009). Aleatory or epistemic? does it matter? Structural Safety 31(2), 105-112.

Esposito, S. (2011). Systemic seismic risk analysis of gas distribution networks.

Jayaram, N. \& J. Baker (2010). Probabilistic seismic lifeline risk assessment using efficient sampling and data reduction techniques. $\mathrm{Ph}$. D. thesis, Stanford University.

Mackie, K. \& B. Stojadinovic (2001). Probabilistic seismic demand model for california highway bridges. Journal of Bridge Engineering 6(6), 468-481.

Moehle, J. \& G. G. Deierlein (2004). A framework methodology for performance-based earthquake engineering. In Proceedings of the 13th World conference on earthquake engineering, pp. 3812-3814. 